Review Article

\title{
Oxidative Stress and Acute Kidney Injury in Critical Illness: Pathophysiologic Mechanisms-Biomarkers-Interventions, and Future Perspectives
}

\author{
Paraskevi Pavlakou, ${ }^{1}$ Vassilios Liakopoulos, ${ }^{2}$ Theodoros Eleftheriadis, ${ }^{3}$ Michael Mitsis, ${ }^{4}$ and \\ Evangelia Dounousi ${ }^{1}$ \\ ${ }^{1}$ Department of Nephrology, Medical School University of Ioannina, Ioannina, Greece \\ ${ }^{2}$ Division of nephrology and Hypertension, 1st Department of Internal Medicine, AHEPA Hospital, School of Medicine, \\ Aristotle University of Thessaloniki, Thessaloniki, Greece \\ ${ }^{3}$ Department of Nephrology, Medical School, University of Thessaly, Larissa, Greece \\ ${ }^{4}$ Department of Surgery, Medical School University of Ioannina, Ioannina, Greece
}

Correspondence should be addressed to Evangelia Dounousi; evangeldou@gmail.com

Received 15 May 2017; Revised 9 August 2017; Accepted 20 August 2017; Published 28 September 2017

Academic Editor: Rodrigo Franco

Copyright (c) 2017 Paraskevi Pavlakou et al. This is an open access article distributed under the Creative Commons Attribution License, which permits unrestricted use, distribution, and reproduction in any medium, provided the original work is properly cited.

\begin{abstract}
Acute kidney injury (AKI) is a multifactorial entity that occurs in a variety of clinical settings. Although AKI is not a usual reason for intensive care unit (ICU) admission, it often complicates critically ill patients' clinical course requiring renal replacement therapy progressing sometimes to end-stage renal disease and increasing mortality. The causes of AKI in the group of ICU patients are further complicated from damaged metabolic state, systemic inflammation, sepsis, and hemodynamic dysregulations, leading to an imbalance that generates oxidative stress response. Abundant experimental and to a less extent clinical data support the important role of oxidative stress-related mechanisms in the injury phase of AKI. The purpose of this article is to present the main pathophysiologic mechanisms of AKI in ICU patients focusing on the different aspects of oxidative stress generation, the available evidence of interventional measures for AKI prevention, biomarkers used in a clinical setting, and future perspectives in oxidative stress regulation.
\end{abstract}

\section{Introduction}

Acute kidney injury (AKI) is a multifactorial clinical entity that presents with primary and secondary nonspecific manifestations due to a variety of causes (Table 1). Until the beginning of the 21st century, the incidence of AKI was not accurately reported due to the fact that AKI definition was highly dependent on clinician's opinion and widely varied among different centers [1]. The definition and diagnosis of AKI based on standard criteria were first developed in 2004 by the Second International Consensus Conference of the Acute Dialysis Quality Initiative (ADQI) Group which introduced the RIFLE (Risk, Injury, Failure, Loss, End-stage kidney disease) criteria [2] (Table 1). The different stages in RIFLE classification are delineated according to changes in serum creatinine levels and/or glomerular filtration rate (GFR) or urine output [2]. In 2007, the Acute Kidney Injury Network (AKIN) published a report that established AKI is the term to be used in order to describe the whole spectrum of acute kidney failure and proposed a modified RIFLE classification without including separately renal replacement therapy (RRT) [3]. Most recently, in 2012, Kidney Disease: Improving Global Outcomes (KDIGO) working group proposed that for accuracy purposes, serum creatinine measurements should be used instead of GFR estimation when staging AKI [4] and a guideline report 
TABLE 1: Common causes and susceptibilities for AKI.

\begin{tabular}{l}
\hline Sepsis \\
Circulatory compromise (shock) \\
Burns/trauma \\
Cardiac surgery (especially with cardiopulmonary bypass) \\
Major (noncardiac) surgery \\
Nephrotoxic drugs \\
Radiocontrast agents \\
Poisonous plants/animals \\
Volume depletion \\
Advanced age \\
Female gender \\
Black race \\
Chronic kidney disease \\
Diabetes mellitus \\
Cancer \\
Anemia
\end{tabular}

was endorsed by the National Kidney Foundation Kidney Disease Outcomes Quality Initiative (NKF-KDOQI) as well [5] (Table 2).

AKI represents a major public health problem with a reported incidence of $0.25 \%$ in the general population and $18 \%$ in the hospitalized patients [5]. Although AKI is not a usual cause for admission to intensive care unit (ICU), it often complicates critically ill patients' clinical course. Epidemiologic evidence of all-cause AKI incidence in ICU patients widely varies due to the remarkable polyphony previously used in diagnostic criteria and ranges from 5.7\% [6] to 36\% [7-9]. The severity of AKI defined by RIFLE classification has been reported to be $36.1 \%$ and seems to be an independent risk factor for patients' outcome and mortality $[7,8]$. Moreover, in a critical care setting, AKI is connected with prolongation of hospitalization and need for RRT and occasional progress to chronic kidney disease. Sepsis is the leading cause of AKI in severely ill patients in ICU, accounting for nearly $50 \%$ of cases [10], while common concurrent diseases further complicate the outcome of these patients including congestive heart failure, liver disease, malignancies, and chronic obstructive pulmonary disease [11] as well as preventable causes that are derived from surgical procedures and prolonged hospitalization [12].

Kidney is a highly vulnerable organ, and the etiology of AKI is of multiple origins. Nevertheless, in the majority of situations, renal parenchyma integrity is disrupted either in terms of hypoperfusion that ends up in renal tubular dysfunction or by direct damage from "toxins" that further injure kidney's interstitial tissue and cellular functions [13]. Oxidative stress gives rise to a chain-like response through direct production of reactive oxygen species (ROS) and metabolic products that act as ligands for receptor types (i.e., tolllike receptors) whose activation is the "alarm" for an ongoing harmful process in AKI. Those circulating "toxins" are inflammatory products that mediate the expansion of injury and hemodynamic imbalance [14]. Critical illness is interwoven with acute inflammation and the consequent production of ROS that feed oxidative stress response. Albeit etiology (hemodynamic dysregulations, infections, rhabdomyolysis, cardiorenal syndrome, uremia, inadequate clearance of metabolism products, etc.), AKI and oxidative stress preserve a bidirectional relationship in critically ill patients. The purpose of this article is to present the main pathophysiologic mechanisms of AKI in ICU patients focusing on the different aspects of oxidative stress generation, the available evidence of interventional measures for AKI prevention, biomarkers used in a clinical setting, and future perspectives in oxidative stress regulation.

1.1. Oxidative Stress and Its Pathogenetic Role in AKI. Oxidative metabolism constitutes a fundamental process for aerobic organisms in order to cover energy needs and respond to emergency metabolic situations $[15,16]$. Under normal circumstances, the balance between oxidants and antioxidant production is retained in favor of homeostasis. Oxidative stress was introduced for the first time by Stahland and Sies in 1985 [17] and is briefly defined as the metabolic disturbances, such as increased production of oxidants that leads to the depletion of endogenous antioxidants with inadequate decompensation and ends up in cellular damage [15], dysfunction of proteins, and damage of DNA, lipids, and enzymes [17]. The quantification of oxidative stress can be approached only indirectly, by measuring by-products such as isoprostanes [18], malondialdehyde levels [19], and other protein damage markers [20] with techniques and results that have been questioned. On the other hand, endogenous antioxidant systems are self-defense mechanisms with a crucial participation in the maintenance of immune system integrity that are activated when oxidative stress cannot be counterbalanced [21]. When organisms sense a possible threat, they have the ability to delay metabolic processes and even enter cell cycle arrest in order to avoid further oxidative damage.

The pathophysiology of AKI constitutes a complex interplay among vascular, tubular, and inflammatory factors which is followed by a repair process that can either restore epithelial cells and physiological function or result in progressive fibrosis and chronic kidney damage. Abundant experimental and to a less extent clinical data support the important role of oxidative stress-related mechanics in the injury phase of AKI (Figure 1). The more extensively explored and better-established mechanisms of oxidative stress involved in the pathogenesis of AKI will be reviewed.

1.1.1. Reactive Oxygen Species (ROS) and Nitric Oxide (NO). Mitochondrion is the primary energy factory of the human body and is abundant in proximal renal tubule making renal cortex a crucial field of oxygen use for energy production. Moreover, in AKI, mitochondrial injury precedes other renal manifestations even the increase of serum creatinine levels [22]. The main source of ROS generation is the reduction of oxygen by cytochrome oxidase in mitochondrial electron chain transport (ETC) that results in the production of hydrogen peroxide $\left(\mathrm{H}_{2} \mathrm{O}_{2}\right)$, superoxide anion radical $\left(\mathrm{O}_{2}^{-}\right)$, and hydroxyl radical ( $\mathrm{HO})$ [15]. There is no specific target 
TABLE 2: Acute kidney injury stratification criteria.

\begin{tabular}{|c|c|c|c|c|}
\hline \multirow{2}{*}{$\begin{array}{l}\text { AKIN } \\
\text { Serum creatinine }\end{array}$} & \multirow[b]{2}{*}{ Stage } & \multicolumn{3}{|c|}{ KDIGO } \\
\hline & & Stage & Serum creatinine & Urine output \\
\hline $\begin{array}{l}\geq 0.3 \mathrm{mg} / \mathrm{dL} \text { increase or } \\
\text { increase } \times 1.5-2 \text { from baseline }\end{array}$ & 1 & 1 & $\begin{array}{l}\times 1.5-1.9 \text { from baseline } \\
\text { or } \geq 0.3 \mathrm{mg} / \mathrm{dL} \text { increase }\end{array}$ & $<0.5 \mathrm{~mL} / \mathrm{min} / \mathrm{kg} \times 6-12 \mathrm{~h}$ \\
\hline Increase $\times 2-3$ from baseline & 2 & 2 & $\times 2-2.9$ from baseline & $<0.5 \mathrm{~mL} / \mathrm{min} / \mathrm{kg}$ for $\geq 12 \mathrm{~h}$ \\
\hline $\begin{array}{l}\text { Increase }>\times 3 \text { from baseline or } \\
\text { sCreatinine } \geq 4 \mathrm{mg} / \mathrm{dL} \text { with } \\
\text { acute increase of at least } 0.5 \mathrm{mg} / \mathrm{dL}\end{array}$ & 3 & 3 & $\begin{array}{l}\times 3 \text { from baseline or sCreatinine } \\
\geq 4 \mathrm{mg} / \mathrm{dL} \text { or renal replacement } \\
\text { therapy or eGFR }<35 \mathrm{~mL} / \mathrm{min} / 1.73 \mathrm{~m}^{2} \\
\text { in patients }<18 \text { yo }\end{array}$ & $\begin{array}{c}<0.3 \mathrm{~mL} / \mathrm{min} / \mathrm{kg} \text { for } \geq 24 \mathrm{~h} \text { or } \\
\text { anuria for } \geq 12 \mathrm{~h}\end{array}$ \\
\hline
\end{tabular}

AKIN: Acute Kidney Injury Network; KDIGO: Kidney Disease: Improving Global Outcomes; GFR: glomerular filtration rate; ESKD: end-stage kidney disease.

(i) ROS generation

(ii) NO depletion

(iii) DAMP generation and TLR activation

(iv) Autophagy (mitophagy)

(v) Microvascular dysfunction

(i) Cardiac surgery

(ii) Major trauma

(iii) Decompensated heart failure

(iv) Chronic obstructive pulmonary disease

(v) Sepsis

(vi) Oncohematological malignancies
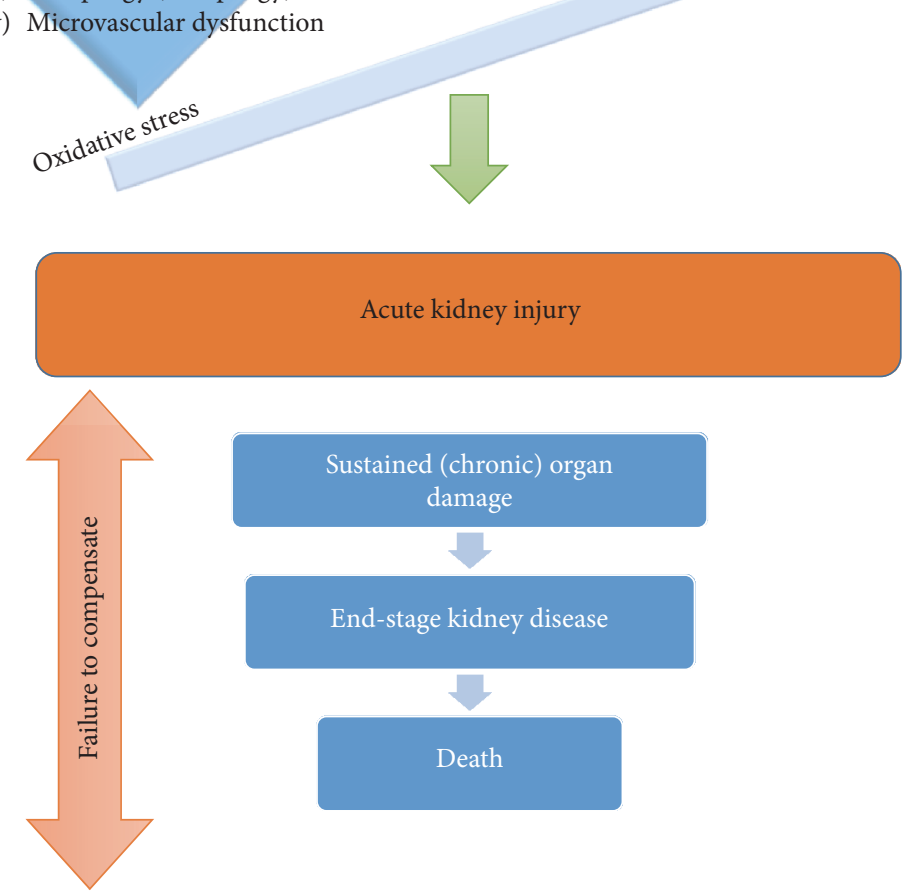

(i) Regulatory mechanisms

(ii) Endogenous antioxidants

Homeostasis maintenance

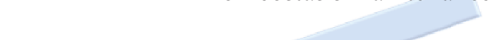


for ROS, but the attack on lipids, proteins, and amino acids results in the formation of unstable molecules that act as radicals and finally convert into compounds with multiple metabolic effects [16]. Consequently, lipid, protein, and nucleic acid peroxides belong in ROS family. Minor ROS generators (about $10 \%$ in total) are xanthine oxidase, NADPH oxidase complex (Nox), and adrenaline/epinephrine as well $[15,23]$.

Kidney receives about $25 \%$ of total blood supply and is rich in mitochondria that render it susceptible to damage from ROS and subsequent development of AKI [24]. Cellular apoptosis, lipid peroxidation, and imbalanced calcium concentration are few of the induced mechanisms by ROS [25]. Two characteristic representatives of AKI are ischemia reperfusion injury (IRI) and sepsis [24]. Aggressive fluid resuscitation for retaining hemodynamic balance may have adverse effects on renal function due to hemodilution and diminished oxygenation [25] but can be prevented through individualization and continuous therapy reassessment [26]. On the contrary, excessive oxygenation that leads to hyperoxia has been linked with further enhancement of ROS production and oxidative stress in patients with acute lung injury [27] and systemic inflammatory response (SIRS) [28].

The endothelial isoform of nitric oxide synthase (eNOS) is the main source of $\mathrm{NO}$ production from arginine and oxygen that is essential for the normal endothelial function and vascular tone, the prevention of platelet aggregation, and presenting anti-inflammatory properties $[16,25]$. The "uncoupling" phenomenon is met when eNOS is deprived of its cofactors (i.e., calmodulin and tetrahydrobiopterin) and results in the oxidation of oxygen and the release of superoxide [29] that acts as a free radical adding on to oxidative stress. The above phenomenon takes place in inflammatory situations (such as sepsis) where there is an incremental cellular NO release (and oxygen consumption) and is mediated by the action of inducible nitric oxide synthase (iNOS). According to a theory, the heterogeneous iNOS expression in AKI that leads to focal increase of NO levels and is further enhanced by microcirculatory dysfunction results in the perpetuation of regional oxygen deprivation [30]. Thus, kidney damage not only is maintained but also expands. The iNOS-dependent inhibition of eNOS deteriorates endothelial function further shaping a triangle among ROS, NO, and oxygen [29, 30] in the pathophysiology of AKI and oxidative stress.

1.1.2. Toll-Like Receptors (TLRs) and Damage-Associated Molecular Pattern (DAMPs). TLRs are transmembrane, pattern recognition receptors, and currently, there have been about 10 recognized subtypes in humans [31]. DAMPs are endogenous molecules that may either initiate immune response or act as proinflammatory mediators (the latest are occasionally called alarmins) [32]. They are presented to the immune system after cellular lysis, scheduled exocytosis, or after the release of enzymes' matrix [33]. Apart from DAMPs, TLRs recognize pathogen-associated molecular pattern (PAMPs) (peptidoglycan and lipopolysaccharide from pathogens). Macrophages, endothelial cells, dendritic cells, and lymphocytes express TLRs. Kidney mesangial and tubular epithelial cells express TLR1, TLR2, TLR3, TLR4, and TLR6. Once a ligand is bind on the receptor, with the complicity of factors such as myeloid differentiation factor 88 (MyD88) and toll-receptor activator of interferon (TRIF), endogenous pathways are activated (nuclear factor kappa-B and mitogen-activated protein kinase pathway) and result in inflammation and interferon production [31, 34].

In 1994, Matzinger introduced the theory of "danger" that is sensed by the immune system, it does not necessarily originate from pathogens, and it has the ability to enhance or fire innate immune response so that the threat is sufficiently defeated [35]. DAMPs are the triggering factors for this process and come from endogenous, damaged cells, usually including proteins. In AKI, heat shock proteins (HSPs) and high-mobility group box-1 (HMGB-1) protein are the most common but several others have been suggested as well $[33,36]$. According to accumulating evidence during oxidative stress, TLR activation from DAMPs further enhances the incremental release of the latest as it was shown with HSp70 and TLR2/TLR4 in an animal model during IRI [37]. On the contrary, the origin of HMGB-1 is not that clear. Evidence from in vitro studies in hypoxic hepatocytes is in favor of ROS regulation on HMGB-1 release with prerequisite functional TLRs [38]. As derived from the aforementioned evidence, there is an ambiguous relationship between DAMPs and TLR activation in oxidative stress. Also, the release of DAMPs is partly determined by TLRs who are the main regulators of overall immune answer in oxidative stress [33]. The magnitude of inflammation-oxidative stress complexity is yet to be revealed and translated.

1.1.3. Autophagy in AKI. Autophagy is a continuous, catabolic process conserved through evolution that takes place at a cellular level [39]. It is generally described as a "housekeeping" process and aims at the removal of damaged and dysfunctional molecules as well as at the enhanced response to acute situations such as nutrient deficiency, ensuring the recycling of components for protein and energy synthesis and the elimination of toxic material [40]. Fundamental for the initiation of autophagy is the expression of the autophagy-related genes (ATG) that were first discovered in yeast, with the produced proteins being subjected to multiple posttranslational modifications that regulate the final outcome [41]. According to evidence from animal models and clinical trials, the ATG proteins increase in AKI. In particular, ATG proteins that augment in AKI with tubular dysfunction are microtubule-associated protein light chain 3 (LC3) and Beclin-1 $[42,43]$. The first step is the formation of an intracellular, double-membrane organelle called phagophore that after the sequestration of the target turns into autophagosome and with the subsequent lysosomal fusion becomes the autolysosome that with the intermediary action of lysosomal enzymes will lead to the degradation of the contained cytoplasmic components in order to provide matrix for "recycling" [44]. The process is complete after lysosomal reformation and the inhibitory effect on autophagy of mammalian target of rapamycin receptor (mTOR) [39, 40]. Nevertheless, there are pending issues regarding the further 
clarification of the complicated signaling pathways in autophagy, their selectivity, and regulation [39].

In AKI, hypoxic damage in tubular epithelial cells is a potent stimulus for autophagy [45] that is generally considered beneficial and nephroprotective, preventing further structural compromise, especially at the S3 segment of the proximal tubule that is vulnerable to oxygen deprivation [46]. In AKI, apart from hypoxia, the increased ROS production due to inflammation and oxidative stress causes mitochondrial depolarization and dysfunction that through the PINK1/Parkin (PTEN-induced putative kinase protein 1) and the BNIP3/NIX/FUNDC1 pathway lead to selective mitochondrial autophagy ("mitophagy") $[24,44]$. Contradictory opinions exist and claim that autophagy can be deleterious promoting cellular apoptosis, adding on to the renal injury [47-49].

1.1.4. Microvascular Dysfunction. Under normal circumstances, outer medulla is perfused with about half blood flow compared to cortex and the consequent partial oxygen pressure is $10-20 \mathrm{mmHg}$ and $50 \mathrm{mmHg}$ [50]. Thus, outer medulla is an especially vulnerable zone to circulatory disturbances and hypoxia. During AKI, the sustained renal perfusion through normal blood flow from renal artery does not secure the unhampered function of the complex renal microvasculature. Evidence data prove the existence of focal hypoxemic renal tissue in AKI [51] that add on to our comprehension regarding the pathophysiology of AKI [52]. In oxygen deprivation, anaerobic glycolysis is enhanced, lactic acid is accumulated, mitochondrial dysfunction is enhanced, and production of ROS and superoxide is upregulated [53]. The injury expands after reperfusion that is characterized by inflammatory response with leukocyte and complements activation that progresses to an oxidant environment that cannot be counterbalanced by antioxidant mechanisms [54] and uneventfully leads to excessive cell death [53].

Endothelium holds a crucial role regarding the expansion of inflammation, through expression of adhesion molecules such as selectins [55], the intracellular adhesion molecule-1 (ICAM-1) [56], and CX3CL1 (fractalkine) [57] that regulate inflammatory cell recruitment. The effect on vascular wall, along with the partly specified changes on glycocalyx [58], is increased permeability that in $\mathrm{AKI}$, is expressed as proteinuria $[58,59]$.

1.2. Prediction of AKI by Oxidative Stress Biomarkers in Critically Ill Patients. A number of obstacles have hampered the investigation of the role of oxidant injury in multiple organ failure and AKI in critically ill patients. Among them is the fact that oxidative stress might be a focal, instant response resulting to the lack of stable, specific oxidative stress biomarkers that can be measured accurately and noninvasively in these patients [16]. Nevertheless, prevention of AKI requires among others the recognition of high-risk patients and early diagnosis based on accurate predictive tools. After the recognition of serum creatinine inadequacy in the prediction of AKI due to the variability of measured levels (based on age, gender, race, and muscle mass) with low sensitivity and specificity [60], novel plasma and urine biomarkers have been introduced. In the meantime, along with the enhanced comprehension of novel biomarker characteristics (for details refer to [61-65]), there is accumulating evidence concerning the predictive value and the clinical applicability of these molecules.

Oxidative stress can be assessed by indirect methods which can measure the stable by-products of ROS activity on biomolecules. In the setting of critical illness, the most commonly measured markers of oxidative stress are isoprostanes, hydroxynonenal and lipid peroxides, chlorinated compounds, oxidized glutathione, nitrated and oxidized proteins, and malondialdehyde detected as thiobarbituric acid reactants (TBARs) [15]. Among them, some biomarkers have been investigated in order to predict the occurrence of AKI in severely ill patients with different results. In an observational cohort study in ICU patients with severe sepsis, Ware et al. found that plasma levels of F2-isoprostanes and isofurans were associated with acute hepatic, coagulation, and renal failure [66]. Liver-type fatty acid-binding protein (L-FABP) has been considered as an important cellular antioxidant during oxidative stress by maintaining low levels of free fatty acids in the cytoplasm of tubular cells through facilitation of intracellular metabolism and excretion in urine. In a number of studies, urine L-FABP has been able to reliably predict the occurrence of AKI and death in ICU patients [64]. Recently, Costa et al. found that erythrocyte superoxide dismutase (SOD1) activity could play a role as an early marker of septic $\mathrm{AKI}$ and could be seen as a new research avenue in the field of biomarker in AKI [67].

According to robust evidence, an AKI-specific biomarker is the neutrophil gelatinase-associated lipocalin (NGAL) that can be measured in both plasma and urine $[68,69]$. NGAL is a multifaceted protein that is rapidly induced and released from the injured distal nephron-among others. It has the ability to scavenge iron whose role is crucial for bacterial survival and is an important component to free radical generation. Thus, NGAL levels have been implicated in various types of organ injury, including myocardial infarction, cancer, sepsis, and AKI [70]. Apart from bacteriostatic effects [71], the protection against oxidative stress damage has been suggested [71, 72], while the exact antioxidative mechanisms of NGAL are still under question. There are data in favor of the upregulation of endogenous antioxidants such as SOD1 and SOD2 as well as HO1 levels [73, 74]. According to a 2009 meta-analysis in 8500 critically ill patients, the area under the curve (AUC) for the prediction of AKI (12 hours earlier) reached 0.85 for plasma NGAL and 0.86 for urine NGAL, superior to the predictive value of serum creatinine levels and eGFR, with sensitivity of $81-96 \%$ and specificity of 51-68\% [75]. Urinary kidney injury molecule-1 (KIM-1) and interleukin-18 (IL-18) are suggested as good markers for the prediction of progressive AKI [75]. The high diagnostic value of IL-18 in AKI (odds ratio (OR): 5.11, AUC: 0.77) [76] is not corroborated by equal prognostic significance in critically ill patients [77], and the careful interpretation of urine IL-18 levels is highly recommended. KIM-1 was attributed with a good prognostic value of AKI development after cardiac surgery with high sensitivity $92-100 \%$ and AUC 0.78-0.91 [78], while the persistent elevation of urine KIM- 
1 levels might correlate with poor prognosis [79]. What should be mentioned is that NGAL, IL-18, and KIM-1 are inflammatory mediators that increase in inflammatory situations regardless of the presence of AKI and are indivisible parameters concerning their assessment in the prediction of AKI [65].

Recently, tissue inhibitor of metalloproteinase 2 (TIMP-2) and insulin-like growth factor-binding protein 7 (IGFBP-7) have been investigated as predictive urine biomarkers of AKI in high-risk patients [80, 81]. Both TIMP-2 and IGFBP-7 are cell cycle arrest biomarkers as they have been implicated in the G1 cell cycle arrest phase during the very early stages of cellular stress. It has been shown that renal tubular cells go through this G1 cell cycle arrest phase following stress due to a number of different causes. Specifically, the SAPPHIRE study assessed the urine product of TIMP-2 and IGFBP-7 and concluded that it is superior in the prediction of KDIGO stage 2-3 AKI compared with the rest of the biomarkers of the study, even NGAL and KIM-1 $(p<0.002)$ in critically ill patients [80]. Further analysis in the SAPPHIRE and OPAL study cohorts has set cut-off values for risk stratification of AKI with high-risk patients when TIMP- $2 \times$ IGFBP-7 is over 0.3 and the highest risk for patients with product value is over 2 [81]. Nevertheless, in persisting AKI that is equal with the ongoing damage, the levels of TIMP-2 $\times$ IGFBP-7 product remain elevated indicating the maintenance of cell cycle arrest (in G1 phase) that may uneventfully lead to failure of recovery and renal fibrosis [82]. Thus, the potential selective intervention in the activation and disruption of cell cycle might be beneficial for renal protection.

\subsection{Clinical Evidence in AKI Prevention by Targeting} Oxidative Stress. Albeit evidence for the role of oxidative stress in the pathogenesis of AKI originating mainly from experimental models and distinctive pathways remains obscure, the idea that controlling oxidative stress in patients with AKI may prevent or attenuate the severity of cellular injury has been explored in the clinical setting. Existing clinical evidence in this field, regarding critically ill and ICU patients, comes from small cohorts and studies. Nevertheless, the scavenging of free radicals in order to avoid the provocation of chain reactions that will lead to regional or generalized oxidative stress demonstrates great interest.

Anesthetics have been suggested as potential oxidative stress scavengers and in particular SOD mimetics (sodium pentothal and propofol) and lidocaine, when used in critical care practice [83]. N-acetylcysteine (NAC) as shown by in vitro studies acts as a direct scavenger of $\mathrm{OH}^{-}$mainly, but when administered orally, the bioavailability is low and even untraceable. The antioxidant action of NAC is mediated by the induction of glutathione synthesis [84]. Data from trials in humans imply that NAC reduces the incidence of AKI after contrast media administration $(p=0.02)$ [85], but the direct intravenous administration of glutathione has been shown to be superior as regards renal protection against contrast-induced nephropathy compared to NAC per os [86].

Apart from the first-line treatment in lipid-lowering therapy, HMG-CoA reductase inhibitors, globally known as statins, participate further in vascular endothelium function preservation through upregulation of eNOS, thus increasing the available NO and contribute to the restriction of free radical generation from lipids' oxidation $[87,88]$. In this direction, results from cohort studies concerning severe illness are in favor of the benefits of statins in the protection of renal function after percutaneous coronary angiography [89], acute coronary syndrome [90], and IRI [91]. On the contrary, a Cochrane database meta-analysis on the prevention of AKI with statin administration prior to major surgery failed to show reduction in AKI incidence for critically ill patients undergoing surgery with cardiac bypass [92].

Ischemic preconditioning (IPR) was introduced in 1986 by Murry et al. in an animal model that sustained brief ischemic episodes before a major ischemic event and resulted in a beneficial outcome for the organ [93]. In 1993, Przyklenk et al. described a slightly different model of ischemic preconditioning (remote and rIPR) [94] that has been further modified and is currently followed, when the direct approach to the involved organ is not feasible. The underlying mechanisms are notably complex and not totally unraveled. In brief, after the main stimuli (ischemia) is withdrawn, a series of responses take place (neural, humoral pathway, and systemic anti-inflammatory response) with the final receiver being the mitochondrion [95]. The subsequent opening of the ATPdependent mitochondrial potassium channel prevents the opening of the mitochondrial permeability transition pores (MPTP) that enhances the stability of its membrane [96] and the survival after IRI [97].

Generally speaking, rIPR concerns clinical practice and especially critical care when it comes to scheduled procedures that carry a significant burden for homeostasis and are closely related with the induction of systematic inflammation and oxidative stress, such as cardiac surgery procedures. The highly vulnerable to hemodynamic imbalance renal cortex and its complex microvasculature are affected by rIPR. According to a recent review (2016) by Ho et al., who included 17 clinical trials that examined the renal outcome in different rIPR cases, a notable renal protection is shown in $12 / 17$ of the trials with no significant deviations in the rest of the trials [98].

1.4. Therapeutic Interventions and Future Perspectives. In the current clinical practice, there is a lack of standardized preventive measures against AKI in severely ill patients apart from general suggestions for maintenance of fluid and electrolyte balance, avoidance of unnecessary exposure to potentially nephrotoxic agents, and continuous clinical monitoring $[99,100]$. Earlier efforts to show benefit in renal outcome in critical care setting through administration of low-dose dopamine in continuous infusion have shown a temporary benefit in urine output [101], but with no significant protection against the development of AKI, the prevention of RRT, and mortality according to meta-analyses [102-104]. In the same patient group, fenoldopam seems to be superior compared to dopamine in the improvement of serum creatinine levels when renal dysfunction is present [105] and according to a meta-analysis in 1290 patients, 
fenoldopam administration reduced the need for RRT support and intensive care unit hospitalization [106].

As regards interventional measures in order to control oxidative stress response in critically ill patients, the interest has been focused on macro- and micronutrients and the correlation of their levels with patients' general clinical course and outcome and not just with the prevention or therapy of AKI. The early recommencement of enteral versus parenteral feeding in ICU patients (even before 48 hours of hospitalization) that contributes on the maintenance of normal intestinal microflora has been correlated with better survival and less infections [107]. Nevertheless, the optimal dose that permits autophagy and provides the highest benefit for ICU patients has not been quantified yet [107, 108]. The supplementation of trace elements in critically ill patients has employed investigators and in particular the administration of thiamine, vitamin $\mathrm{C}$ and $\mathrm{E}$, and selenium separately has been found to improve survival and reduce infectious complications [109]. On the contrary, no clear benefit on critically ill patients' survival was shown in a meta-analysis of 4 trials with zinc administration, neither a benefit in the duration of ICU stay [110]. In a meta-analysis of 21 randomized control trials, it was shown that the supplementation of trace elements correlated with reduction in the number of days with need for mechanical ventilation, but the establishment of clear conclusions regarding the best possible route of administration (enteral versus intravenous) was not feasible due to significant heterogeneity of the available data [111]. Even if this replenishment concerns relatively short periods, toxicity $[109,110]$ is to be kept in mind and appropriate measures should be applied in order to avoid it. In general, the substitution of more than $66 \%$ of the recommended daily allowance of vitamins $\mathrm{A}, \mathrm{C}$, and $\mathrm{E}$ has been shown to improve antioxidant capacity [112]. REDOXS (Reducing Deaths due to Oxidative Stress) study is a blinded randomized trial in 1223 critically ill patients that failed in meeting its original rationale and concluded that the administration of antioxidants and glutamine increased mortality [113]. Among the possible reasons are the doses chosen of the implemented therapeutic strategy and the potential toxicity that may have defined the final outcome [114]. Enteral administration of melatonin [115] and parenteral administrations of NAC plus deferoxamine [116] have been correlated with better total antioxidant capacity (TAC) in serum. As derived from the presented data, we are not yet capable to reach safe conclusions with clinical applicability as regards the initiation, dose, route, and duration of therapy for the aforementioned strategies.

As regards future perspectives, antioxidants targeted to mitochondria have been developed and the main axis of their action is through the electrical potential and the $\mathrm{pH}$ gradient of the mitochondrion that leads to the selective accumulation of these cationic molecules. Mito-vitE, MitoQ, MitoPBN, and MitoPeroxidase have the ability to prevent ROS generation and enhance mitochondrial survival [117-119]. The optimization of understanding the mechanisms of action has gained a lot of interest, as well as the enhancement of their chemical synthesis [120]. Unfortunately, current literature lacks in vitro or in vivo studies investigating the administration of antioxidant targeted molecules.

\section{Conclusions}

Acute kidney injury is a multifactorial clinical entity representing a major health problem. In critical care, AKI remains highly prevalent, complicating the clinical course of patients, extending the need for ICU hospitalization, requiring RRT, and carrying high mortality. Pathogenesis of AKI is complex and remains incompletely elucidated. Oxidative stress is involved in the pathogenesis of AKI and is characterized by complex, codependent mechanisms that progress to organ response and damage. More extensively, main experimentally explored mechanisms of oxidative stress involved in AKI summarize to ROS generation, NO depletion, DAMP generation and TLR activation, autophagy, and microvascular dysfunction. These mechanisms prevail over endogenous antioxidants and regulatory mechanisms so that physiological homeostasis is abolished and AKI is finally installed.

Prevention of AKI is essential and requires among others the recognition of high-risk patients and early diagnosis based on accurate predictive tools. In the setting of critical illness, the most commonly measured markers of oxidative stress are isoprostanes, hydroxynonenal and lipid peroxides, chlorinated compounds, oxidized glutathione, nitrated and oxidized proteins, and TBARs. Novel AKI-specific biomarkers available are NGAL, KIM- 1 , and levels of TIMP- $2 \times$ IGFBP-7 with accumulating evidence being in favor of their diagnostic and prognostic value. Further progress that will encompass in daily practice techniques allowing more accurate assessment of oxidative stress will further improve the prevention of AKI in critical care. Therapeutic interventions trying to control oxidative stress response in critically ill patients have been focused on macro- and micronutrients. Currently, there are encouraging results from the inhibition of oxidative stress via exogenous administration of antioxidants and methods as ischemic preconditioning, but no standardized therapeutic protocols exist. The role of antioxidant therapy requires further elucidation and attention in the care of critically ill patients and in AKI. The meticulous study and interpretation of available observational data and expansion of existing knowledge through well-designed interventional studies in the setting of critical illness are necessary.

\section{Conflicts of Interest}

The authors declare that there is no conflict of interest regarding the publication of this paper.

\section{References}

[1] R. L. Mehta and G. M. Chertow, "Acute renal failure definitions and classification: time for change?," Journal of the American Society of Nephrology, vol. 14, no. 8, pp. 21782187, 2003.

[2] R. Bellomo, C. Ronco, J. A. Kellum, R. L. Mehta, and P. Palevsky, "Acute renal failure - definition, outcome measures, animal models, fluid therapy and information technology needs : the second international consensus conference of 
the acute dialysis quality initiative ( ADQI ) group," Critical Care, vol. 8, no. 4, pp. R204-R212, 2004.

[3] R. L. Mehta, J. A. Kellum, S. V. Shah et al., "Acute kidney injury network: report of an initiative to improve outcomes in acute kidney injury," Critical Care, vol. 11, no. 2, article R31, 2007.

[4] KDIGO clinical practice guidelines for acute kidney injury," Kidney International Supplements, vol. 2, no. 1, 2012.

[5] P. M. Palevsky, K. D. Liu, P. D. Brophy et al., "KDOQI US commentary on the 2012 KDIGO clinical practice guideline for acute kidney injury," American Journal of Kidney Diseases, vol. 61, no. 5, pp. 649-672, 2013.

[6] S. Uchino, J. A. Kellum, R. Bellomo et al., "Acute renal failure in critically ill patients: a multinational, multicenter study," Journal of the American Medical Association, vol. 294, no. 7, pp. 813-818, 2005.

[7] M. Ostermann and R. W. Chang, "Acute kidney injury in the intensive care unit according to RIFLE," Critical Care Madicine, vol. 35, no. 8, pp. 1837-1843, 2007.

[8] S. M. Bagshaw, C. George, I. Dinu, and R. Bellomo, "A multicentre evaluation of the RIFLE criteria for early acute kidney injury in critically ill patients," Neprhology, Dialysis, Transplantation, vol. 23, no. 4, pp. 1203-1210, 2008.

[9] K. Singbartl and J. A. Kellum, "AKI in the ICU: definition, epidemiology, risk stratification, and outcomes," Kidney International, vol. 81, no. 10, pp. 819-825, 2012.

[10] J. Case, S. Khan, R. Khalid, and A. Khan, "Epidemiology of acute kidney injury in the intensive care unit," Critical Care Research and Practice, vol. 2013, Article ID 479730, 9 pages, 2013.

[11] M. P. Le Guen, A. E. Tobin, and D. Reid, "Intensive care unit admission in patients following rapid response team activation: call factors, patient characteristics and hospital outcomes," Anaesthesia and Intensive Care, vol. 43, no. 2, pp. 211-215, 2015.

[12] A. Vlayen, S. Verelst, G. Bekkering, W. Schrooten, J. Hellings, and N. Claes, "Incidence and preventability of adverse events requiring intensive care admission: a systematic review," Journal of Evaluation in Clinical Practice, vol. 18, pp. 485497, 2012.

[13] J. V. Bonventre, "Pathophysiology of AKI: injury and normal and abnormal repair," Contributions to Nephrology, vol. 165, pp. 9-17, 2010.

[14] S. D. Glodowski and G. Wagener, "New insights into the mechanisms of acute kidney injury in the intensive care unit," Journal of Clinical Anesthesia, vol. 27, no. 2, pp. 175-180, 2015.

[15] V. I. Lushchak, "Free radicals, reactive oxygen species, oxidative stress and its classification," Chemico-Biological Interactions, vol. 224, pp. 164-175, 2014.

[16] T. Lemineur, G. Deby-Dupont, and J.-C. Preiser, "Biomarkers of oxidative stress in critically ill patients: what should be measured, when and how?," Current Opinion in Clinical Nutrition and Metabolic Care, vol. 9, no. 6, pp. 704-710, 2006.

[17] W. Stahland and H. Sies, Oxidative Stress, Heinrich-Heine University Düsseldorf, Faculty of Medicine, Institute of Biochemistry \& Molecular Biology, 1985.

[18] D. Milatovic, T. J. Montine, and M. Aschner, "Measurement of isoprostanes as markers of oxidative stress," Methods in Molecular Biology, vol. 758, pp. 195-204, 2011.
[19] B. Antus, O. Drozdovszky, I. Barta, and K. Kelemen, "Comparison of airway and systemic malondialdehyde levels for assessment of oxidative stress in cystic fibrosis," Lung, vol. 193, no. 4, pp. 597-604, 2013.

[20] N. Rabbani, F. Shaheen, A. Anwar, J. Masania, and P. J. Thornalley, "Assay of methylglyoxal-derived protein and nucleotide AGEs," Biochemical Society Transactions, vol. 42, no. 2, pp. 511-517, 2014.

[21] M. De la Fuente, "Effects of antioxidants on immune system ageing," European Journal of Clinical Nutrition, vol. 56, pp. S5-S8, 2002.

[22] K. M. Ralto and S. M. Parikh, "Mitochondria in acute kidney injury," Seminars in Nephrology, vol. 36, no. 1, pp. 8-16, 2016.

[23] I. Peluso, M. Palmery, J. Pérez-Jiménez, and G. Drummen, "Biomarkers of oxidative stress in experimental models and human studies with nutraceuticals: measurement, interpretation, and significance," Oxidative Medicine and Cellular Longevity, vol. 2016, Article ID 6159810, 2 pages, 2016.

[24] A. Sureshbabu, S. W. Ryter, and M. E. Choi, "Oxidative stress and autophagy: crucial modulators of kidney injury," Redox Biology, vol. 4, pp. 208-214, 2015.

[25] U. Aksu, C. Demirci, and C. Ince, "The pathogenesis of acute kidney injury and the toxic triangle of oxygen, reactive oxygen species and nitric oxide," Contributions to Nephrology, vol. 174, pp. 119-128, 2011.

[26] M. Labib, R. Khalid, A. Khan, and S. Khan, "Volume management in the critically ill patient with acute kidney injury," Critical Care Research and Practice, vol. 2013, Article ID 792830, 6 pages, 2013.

[27] M. Lois, B. Las, and M. Moss, "Oxidant-antioxidant balance in acute lung injury," Chest, vol. 122, Supplement 6, pp. 314S-320S, 2000.

[28] D. Bar-Or, M. M. Carrick, C. W. Mains, L. T. Rael, D. Slone, and E. N. Brody, "Sepsis, oxidative stress, and hypoxia: are there clues to better treatment?," Redox Report Journal, vol. 20, no. 5, pp. 193-197, 2015.

[29] T. J. Rabelink and A. J. van Zonneveld, "Coupling eNOS uncoupling to the innate immune response," Arteriosclerosos, Thrombosis and Vascular Biology, vol. 26, no. 12, pp. 25852587, 2006.

[30] H. Gomez, C. Ince, D. De Backer et al., "A unified theory of sepsis-induced acute kidney injury: inflammation, microcirculatory dysfunction, bioenergetics, and the tubular cell adaptation to injury," Shock, vol. 41, pp. 3-11, 2014.

[31] D. De Nardo, "Toll-like receptors: activation, signalling and transcriptional modulation," Cytokine, vol. 74, no. 2, pp. 181-189, 2015.

[32] H. Kono and K. L. Rock, "How dying cells alert the immune system to danger," Nature Reviews Immunology, vol. 8, no. 4, pp. 279-289, 2008.

[33] R. Gill, A. Tsung, and T. Billiar, "Linking oxidative stress to inflammation: toll-like receptors," Free Radical Biology and Medicine, vol. 48, no. 9, pp. 1121-1132, 2008.

[34] P. G. Vallés, A. G. Lorenzo, V. Bocanegra, and R. Vallés, "Acute kidney injury: what part do toll-like receptors play?," International Journal of Nephrology and Renovascular Disease, vol. 7, pp. 241-251, 2014.

[35] P. Matzinger, "Tolerance, danger, and the extended family," Annual Review of Immunology, vol. 12, pp. 991-1045, 1994.

[36] D. L. Rosin and M. D. Okusa, "Dangers within: DAMP responses to damage and cell death in kidney disease," 
Journal of the American Society of Nephrology, vol. 22, no. 3, pp. 416-425, 2011.

[37] B. S. Kim, S. W. Lim, C. Li et al., "Ischemia-reperfusion injury activates innate immunity in rat kidneys," Transplantation, vol. 79, no. 10, pp. 1370-1377, 2005.

[38] A. Tsung, J. R. Klune, X. Zhang et al., "HMGB1 release induced by liver ischemia involves toll-like receptor 4-dependent reactive oxygen species production and calciummediated signaling," Journal of Experimental Medicine, vol. 204, no. 12, pp. 2913-2923, 2007.

[39] Z. Yang and D. J. Klionsky, "Mammalian autophagy: core molecular machinery and signaling regulation," Current Opinion in Cell Biology, vol. 22, no. 2, pp. 124-131, 2010.

[40] P. Duann, E. Lianos, J. Ma, and P.-H. Lin, “Autophagy, innate immunity and tissue repair in acute kidney injury," International Journal of Molecular Sciences, vol. 17, no. 5, p. 662, 2016.

[41] Y. Xie, R. Kang, X. Sun et al., "Posttranslational modification of autophagy-related proteins in macroautophagy," Autophagy, vol. 11, no. 1, pp. 28-45, 2015.

[42] C.-T. Chien, S.-K. Shyue, and M.-K. Lai, "Bcl-xL augmentation potentially reduces ischemia/reperfusion induced proximal and distal tubular apoptosis and autophagy," Transplantation, vol. 84, no. 9, pp. 1183-1190, 2007.

[43] H.-H. Wu, T.-Y. Hsiao, C.-T. Chien, and M.-K. Lai, "Ischemic conditioning by short periods of reperfusion attenuates renal ischemia/reperfusion induced apoptosis and autophagy in the rat," Journal of Biomedical Science, vol. 16, no. 1, p. 19, 2016.

[44] G. P. Kaushal and S. V. Shah, "Autophagy in acute kidney injury," Kidney International, vol. 89, no. 4, pp. 779-791, 2016.

[45] C. Suzuki, Y. Isaka, Y. Takabatake et al., "Participation of autophagy in renal ischemia/reperfusion injury," Biochemical and Biophysical Research Communications, vol. 368, no. 1, pp. 100-106, 2008.

[46] A. Melk, A. Baisantry, and R. Schmitt, "The yin and yang of autophagy in acute kidney injury," Autophagy, vol. 12, pp. 596-597, 2016.

[47] Z. Wang and M. E. Choi, "Autophagy in kidney health and disease," Antioxidants and Redox Signaling, vol. 40, no. 3, pp. 519-537, 2014.

[48] J.-P. Decuypere, L. J. Ceulemans, P. Agostinis et al., “Autophagy and the kidney: implications for ischemia-reperfusion injury and therapy," American Journal of Kidney Diseases, vol. 66, no. 4, pp. 699-709, 2015.

[49] L. He, M. J. Livingston, and Z. Dong, “Autophagy in acute kidney injury and repair," Nephron Clinical Practice, vol. 127, pp. 56-60, 2014.

[50] S.-E. Ricksten, G. Bragadottir, and B. Redfors, "Renal oxygenation in clinical acute kidney injury," Crittical Care, vol. 17, no. 2, p. 221, 2013.

[51] A. Abdelkader, J. Ho, C. P. C. Ow et al., "Renal oxygenation in acute renal ischemia-reperfusion injury," American Journal of Physiology Renal Physiology, vol. 306, pp. F1026-F1038, 2014.

[52] C. Ince, "The central role of renal microcirculatory dysfunction in the pathogenesis of acute kidney injury," Nephron Clinical Practice, vol. 127, no. 1-4, pp. 124128, 2014.
[53] N. Chatauret, L. Badet, B. Barrou, and T. Hauet, "Ischemiareperfusion: from cell biology to acute kidney injury," Progrès en Urologie, vol. 24, Supplement 1, pp. S4-12, 2014.

[54] B. Rovcanin, B. Medic, G. Kocic, T. Cebovic, M. Ristic, and M. Prostran, "Molecular dissection of renal ischemia-reperfusion: oxidative stress and cellular events," Current Medicinal Chemistry, vol. 23, no. 19, pp. 1965-1980, 2016.

[55] R. P. McEver, "Selectins: initiators of leucocyte adhesion and signalling at the vascular wall," Cardiovascular Research, vol. 107, no. 3, pp. 331-339, 2015.

[56] A. M. Witkowska and M. H. Borawska, "Soluble intercellular adhesion molecule-1 (sICAM-1): an overview," European Cytokine Network, vol. 15, no. 2, pp. 91-98, 2004.

[57] W. Liu, L. Jiang, C. Bian et al., "Role of CX3CL1 in diseases," Archivum Immunologiae et Therapiae Experimentalis, vol. 64, no. 5, pp. 371-383, 2016.

[58] A. H. Salmon and S. C. Satchell, "Endothelial glycocalyx dysfunction in disease: albuminuria and altered microvascular permeability," Journal of Pathology, vol. 226, no. 4, pp. 562-574, 2012.

[59] J. W. McCullough, B. Renner, and J. M. Thurman, "The role of the complement system in acute kidney injury," Seminars in Nephrology, vol. 33, no. 6, pp. 543-556, 2013.

[60] P. Dennen and C. R. Parikh, "Biomarkers of acute kidney injury: can we replace serum creatinine?," Clinical Nephrology, vol. 68, no. 5, pp. 269-278, 2007.

[61] N. Kito, K. Endo, M. Ikesue, H. Weng, and N. Iwai, "miRNA profiles of tubular cells: diagnosis of kidney injury," BioMed Research International, vol. 2015, Article ID 465479, 9 pages, 2015.

[62] S. Banaei, "Novel role of microRNAs in renal ischemia reperfusion injury," Renal Failure, vol. 37, no. 7, pp. 1073-1079, 2015.

[63] M. Andreucci, T. Faga, A. Pisani, M. Perticone, and A. Michael, "The ischemic/nephrotoxic acute kidney injury and the use of renal biomarkers in clinical practice," European Journal of Internal Medicine, vol. 39, pp. 1-8, 2016.

[64] S. Kokkoris, C. Pipili, E. Grapsa, T. Kyprianou, and S. Nanas, "Novel biomarkers of acute kidney injury in the general adult ICU: a review," Renal Failure, vol. 35, no. 4, pp. 579-591, 2013.

[65] J. Naud and M. Leblanc, "Biomarkers in acute kidney injury," Kidney International, vol. 3, pp. 115-125, 2011.

[66] L. B. Ware, J. P. Fessel, A. K. May, and L. J. Roberts II, "Plasma biomarkers of oxidant stress and development of organ failure in severe sepsis," Shock, vol. 36, no. 1, pp. 12$17,2006$.

[67] N. A. Costa, A. L. Gut, P. S. Azevedo et al., "Erythrocyte superoxide dismutase as a biomarker of septic acute kidney injury," Annals of Intensive Care, vol. 6, no. 1, p. 95, 2016.

[68] E. Krzeminska, A. Wyczalkowska-Tomasik, N. Korytowska, and L. Paczek, "Comparison of two methods for determination of NGAL levels in urine: ELISA and CMIA," Journal of Clinical Laboratory Analysis, vol. 30, no. 6, pp. 956-960, 2016.

[69] A. Gagneux-Brunon, P. Delanaye, D. Legrand, E. Cavalier, and C. Mariat, "NGAL, biomarqueur de lésion rénale : point d'étape en 2012," Néphrologie \& Thérapeutique, vol. 8, no. 7, pp. 508-515, 2012. 
[70] S. Chakraborty, S. Kaur, S. Guha, and S. K. Batra, "The multifaceted roles of neutrophil gelatinase associated lipocalin (NGAL) in inflammation and cancer," Biochimica et Biophysica Acta (BBA) - Reviews on Cancer, vol. 1826, no. 1, pp. 129-169, 2012.

[71] K. M. Schmidt-Ott, K. Mori, J. Y. Li et al., "Dual action of neutrophil gelatinase-associated lipocalin," Journal of the American Society of Nephrology, vol. 18, no. 2, pp. 407-413, 2007.

[72] M. H. Roudkenar, R. Halabian, Z. Ghasemipour et al., "Neutrophil gelatinase-associated lipocalin acts as a protective factor against $\mathrm{H} 2 \mathrm{O} 2$ toxicity," Archives of Medical Research, vol. 39, no. 6, pp. 560-566, 2008.

[73] P. Bahmani, R. Halabian, M. Rouhbakhsh et al., "Neutrophil gelatinase-associated lipocalin induces the expression of heme oxygenase- 1 and superoxide dismutase 1, 2," Cell Stress and Chaperones, vol. 15, no. 4, pp. 395-403, 2010.

[74] M. Roudkenar, R. Halabian, P. Bahmani, A. Roushandeh, Y. Kuwahara, and M. Fukumoto, "Neutrophil gelatinaseassociated lipocalin: a new antioxidant that exerts its cytoprotective effect independent on heme oxygenase-1," Free Radical Research, vol. 45, pp. 810-819, 2011.

[75] A. Haase-Fielitz, M. Haase, and P. Devarajan, "Neutrophil gelatinase-associated lipocalin as a biomarker of acute kidney injury: a critical evaluation of current status," Annals of Clinical Biochemistry, vol. 51, Part 3, pp. 335-351, 2014.

[76] X. Lin, J. Yuan, Y. Zhao, and Y. Zha, "Urine interleukin-18 in prediction of acute kidney injury: a systemic review and meta-analysis," Journal of Nephrology, vol. 28, no. 1, pp. 716, 2015.

[77] S. Nisula, R. Yang, M. Poukkanen et al., "Predictive value of urine interleukin-18 in the evolution and outcome of acute kidney injury in critically ill adult patients," British Journal of Anaesthesia, vol. 114, no. 3, pp. 460-468, 2015.

[78] Y. Huang and A. C. Don-Wauchope, "The clinical utility of kidney injury molecule 1 in the prediction, diagnosis and prognosis of acute kidney injury: a systematic review," Inflammation \& Allergy-Drug Targets, vol. 10, no. 4, pp. 260-271, 2011.

[79] Y. Tu, H. Wang, R. Sun et al., "Urinary netrin-1 and KIM-1 as early biomarkers for septic acute kidney injury," Renal Failure, vol. 36, no. 10, pp. 1559-1563, 2014.

[80] K. Kashani, A. Al-Khafaji, T. Ardiles et al., "Discovery and validation of cell cycle arrest biomarkers in human acute kidney injury," Critical Care, vol. 17, no. 1, article R25, 2013.

[81] E. A. J. Hoste, P. A. Mccullough, K. Kashani et al., "Derivation and validation of cutoffs for clinical use of cell cycle arrest biomarkers," Nephrology, Dialysis, Transplantation, vol. 29, pp. 2054-2061, 2014.

[82] J. A. Kellum and L. S. Chawla, "Cell-cycle arrest and acute kidney injury: the light and the dark sides," Nephrology, Dialysis, Transplantation, vol.31, no. 1,pp.16-22, 2016.

[83] M. S. Hatwalne, "Free radical scavengers in anaesthesiology and critical care," Indian Journal of Anesthesiology, vol. 56, pp. 227-233, 2012.

[84] S. Fishbane, J. H. Durham, K. Marzo, and M. Rudnick, "Nacetylcysteine in the prevention of radiocontrast-induced nephropathy," Journal of the American Society of Nephrology, vol. 15, no. 2, pp. 251-260, 2004.

[85] M. Ozaydin, T. Peker, S. Akcay et al., "Addition of N-acetyl cysteine to carvedilol decreases the incidence of acute renal injury after cardiac surgery," Clinical Cardiology, vol. 37, no. 2, pp. 108-114, 2014.

[86] T. Saitoh, H. Satoh, M. Nobuhara et al., "Intravenous glutathione prevents renal oxidative stress after coronary angiography more effectively than oral $\mathrm{N}$-acetylcysteine," Heart and Vessels, vol. 26, no. 5, pp. 465-472, 2011.

[87] R. P. Mason, M. F. Walter, and R. F. Jacob, "Effects of HMG$\mathrm{CoA}$ reductase inhibitors on endothelial function: role of microdomains and oxidative stress," Circulation, vol. 109, no. 21, Supplement 1, pp. II-34-II-41, 2004.

[88] V. Lahera, M. Goicoechea, S. G. de Vinuesa et al., "Endothelial dysfunction, oxidative stress and inflammation in atherosclerosis: beneficial effects of statins," Current Medicinal Chemistry, vol. 14, no. 2, pp. 243-248, 2007.

[89] S. Cao, P. Wang, K. Cui, L. Zhang, and Y. Hou, "Atorvastatin prevents contrast agent-induced renal injury in patients undergoing coronary angiography by inhibiting oxidative stress," Journal of Southern Medical University, vol. 32, no. 11, pp. 1600-1602, 2012.

[90] M. Leoncini, A. Toso, M. Maioli, F. Tropeano, S. Villani, and F. Bellandi, "Early high-dose rosuvastatin for contrastinduced nephropathy prevention in acute coronary syndrome," Journal of the American College of Cardiology, vol. 63, no. 1, pp. 71-79, 2014.

[91] S. Sharyo, N. Yokota-Ikeda, M. Mori et al., "Pravastatin improves renal ischemia-reperfusion injury by inhibiting the mevalonate pathway," Kidney International, vol. 74, no. 5, pp. 577-584, 2008.

[92] M. Lewicki, I. Ng, and A. G. Schneider, "HMG CoA reductase inhibitors (statins) for preventing acute kidney injury after surgical procedures requiring cardiac bypass," The Cochrane Database of Systematic Reviews, vol. 3, article CD010480, 2015.

[93] C. E. Murry, R. B. Jennings, and K. A. Reimer, "Preconditioning with ischemia: a delay of lethal cell injury in ischemic myocardium," Circulation, vol. 74, no. 5, pp. 1124-1136, 1986.

[94] K. Przyklenk, B. Bauer, M. Ovize, R. A. Kloner, and P. Whittaker, "Regional ischemic "preconditioning" protects remote virgin myocardium from subsequent sustained coronary occlusion," Circulation, vol. 87, no. 3, pp. 893-899, 1993.

[95] D. J. Hausenloy, "Cardioprotection techniques: preconditioning, postconditioning and remote con-ditioning (basic science)," Current Pharmaceutical Design, vol. 19, pp. 45444563, 2013.

[96] H. Ma, X. Huang, Q. Li, Y. Guan, F. Yuan, and Y. Zhang, "ATP-dependent potassium channels and mitochondrial permeability transition pores play roles in the cardioprotection of theaflavin in young rat," The Journal of Physiological Sciences, vol. 61, no. 4, pp. 337-342, 2011.

[97] N. Gassanov, A. M. Nia, E. Caglayan, and F. Er, "Remote ischemic preconditioning and renoprotection: from myth to a novel therapeutic option?," Journal of the American Society of Nephrology, vol. 25, pp. 216-224, 2014.

[98] P. W.-L. Ho, W.-F. Pang, and C.-C. Szeto, "Remote ischaemic pre-conditioning for the prevention of acute kidney injury," Nephrology, vol. 21, no. 4, pp. 274-285, 2016.

[99] S. M. Bagshaw, R. Bellomo, P. Devarajan et al., "Review article: acute kidney injury in critical illness," Canadian Journal of Anesthesia, vol. 57, no. 11, pp. 985-998, 2010. 
[100] M. L. Bentley, H. L. Corwin, and J. Dasta, "Drug-induced acute kidney injury in the critically ill adult: recognition and prevention strategies," Critical Care Medicine, vol. 38, Supplement 6, pp. S169-S174, 2010.

[101] C. N. Pereira, F. R. Machado, H. P. Guimarães, A. P. R. Senna, and J. L. G. do Amaral, "Hemodynamics and renal function during administration of low-dose dopamine in severely ill patients," São Paulo Medical Journal, vol. 122, no. 4, pp. 141-146, 2004.

[102] J. O. Friedrich, N. Adhikari, M. S. Herridge, and J. Beyene, "Meta-analysis: low-dose dopamine increases urine output but does not prevent renal dysfunction or death," Annals of Internal Medicine, vol. 142, no. 7, pp. 510-524, 2010.

[103] P. Marik, "Low-dose dopamine: a systematic review," Intensive Care Medicine, vol. 28, no. 7, pp. 877-883, 2002.

[104] J. A. Kellum and J. M. Decker, "Use of dopamine in acute renal failure: a meta-analysis," Critical Care Medicine, vol. 29, no. 8, pp. 1526-1531, 2001.

[105] N. Brienza, V. Malcangi, L. Dalfino et al., "A comparison between fenoldopam and low-dose dopamine in early renal dysfunction of critically ill patients," Critical Care Medicine, vol. 34, no. 3, pp. 707-714, 2006.

[106] G. Landoni, G. G. L. Biondi-Zoccai, J. A. Tumlin et al., "Beneficial impact of fenoldopam in critically ill patients with or at risk for acute renal failure: a meta-analysis of randomized clinical trials," American Journal of Kidney Diseases, vol. 49, no. 1, pp. 56-68, 2007.

[107] J. J. Patel, R. T. Hurt, S. A. McClave, and R. G. Martindale, "Critical care nutrition: where's the evidence?," Critical Care Clinics, vol. 33, no. 2, pp. 397-412, 2017.

[108] S. A. McClave and P. J. M. Weijs, "Preservation of autophagy should not direct nutritional therapy," Current Opinion in Clinical Nutrition and Metabolic Care, vol. 18, no. 2, pp. 155-161, 2015.

[109] M. M. Berger and A. Shenkin, "Update on clinical micronutrient supplementation studies in the critically ill," Current Opinion in Clinical Nutrition and Metabolic Care, vol. 9, no. 6, pp. 711-716, 2006.

[110] D. K. Heyland, N. Jones, N. Z. Cvijanovich, and H. Wong, "Zinc supplementation in critically ill patients: a key pharmaconutrient?," Journal of Parenteral and Enteral Nutrition, vol. 32, no. 5, pp. 509-519, 2008.

[111] W. Manzanares, R. Dhaliwal, X. Jiang, L. Murch, and D. K. Heyland, "Antioxidant micronutrients in the critically ill: a systematic review and meta-analysis," Critical Care, vol. 16, no. 2, article R66, 2012.

[112] J. Abilés, A. P. de la Cruz, J. Castaño et al., "Oxidative stress is increased in critically ill patients according to antioxidant vitamins intake, independent of severity: a cohort study," Critical Care, vol. 10, no. 5, article R146, 2006.

[113] D. Heyland, J. Muscedere, P. E. Wischmeyer et al., "A randomized trial of glutamine and antioxidants in critically ill patients," New England Journal of Medicine, vol. 368, no. 16, pp. 1489-1497, 2013.

[114] D. K. Heyland and R. Dhaliwal, "Role of glutamine supplementation in critical illness given the results of the REDOXS study," Journal of Parenteral and Enteral Nutrition, vol. 37, p. 442, 2013.

[115] G. Mistraletti, R. Paroni, M. Umbrello et al., "Melatonin pharmacological blood levels increase total antioxidant capacity in critically ill patients," International Journal of Molecular Sciences, vol. 18, no. 4, p. 759, 2017.

[116] C. M. Fraga, C. D. Tomasi, D. Biff et al., "The effects of $\mathrm{N}$-acetylcysteine and deferoxamine on plasma cytokine and oxidative damage parameters in critically ill patients with prolonged hypotension: a randomized controlled trial," Journal of Clinical Pharmacology, vol. 52, no. 9, pp. 13651372, 2012.

[117] S. S. Sheu, D. Nauduri, and M. W. Anders, "Targeting antioxidants to mitochondria: a new therapeutic direction," Biochimica et Biophysica Acta (BBA) - Molecular Basis of Disease, vol. 1762, no. 2, pp. 256-265, 2006.

[118] A. O. Oyewole and M. A. Birch-Machin, "Mitochondriatargeted antioxidants," The FASEB Journal, vol. 29, no. 12, pp. 4766-4771, 2015.

[119] M. W. Anders, J. L. Robotham, and S.-S. Sheu, "Mitochondria: new drug targets for oxidative stress-induced diseases," Expert Opinion on Drug Metabolism \& Toxicology, vol. 2, no. 1, pp. 71-79, 2006.

[120] V. J. A. Jameson, H. M. Cochemé, A. Logan, L. R. Hanton, R. A. J. Smith, and M. P. Murphy, "Synthesis of triphenylphosphonium vitamin $\mathrm{E}$ derivatives as mitochondriatargeted antioxidants," Tetrahedron, vol. 71, no. 44, pp. 8444-8453, 2015. 


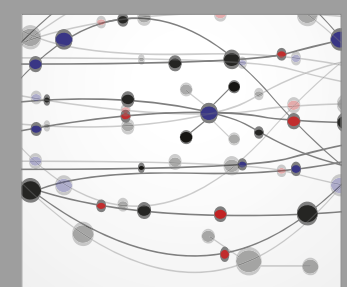

The Scientific World Journal
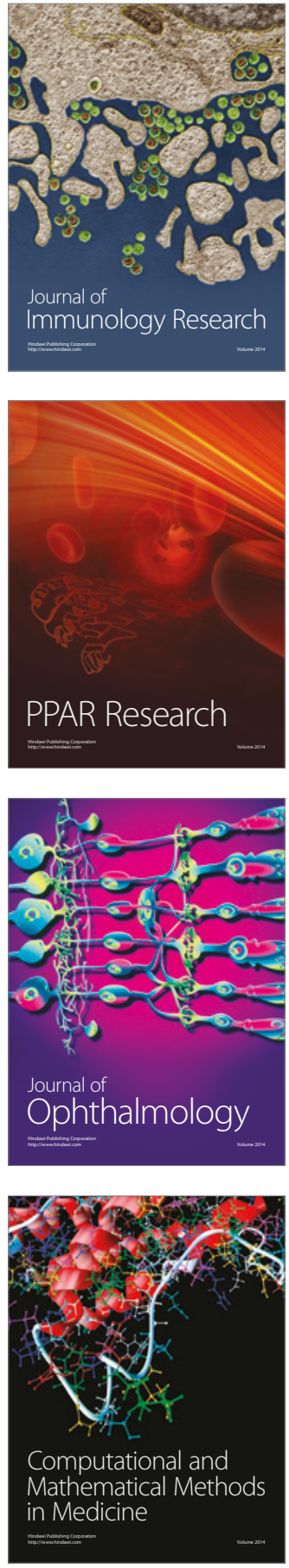

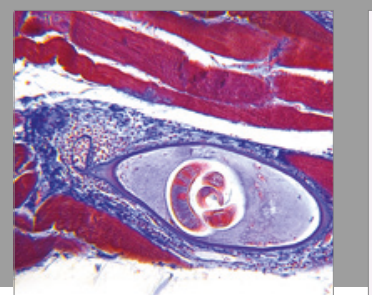

Gastroenterology Research and Practice
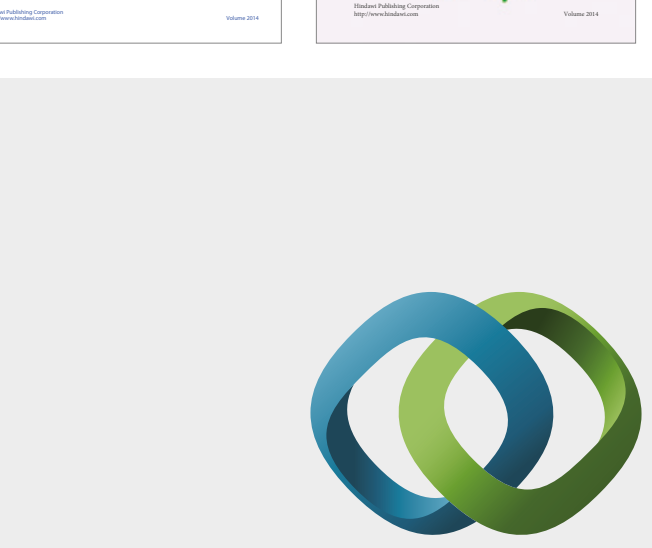

\section{Hindawi}

Submit your manuscripts at

https://www.hindawi.com
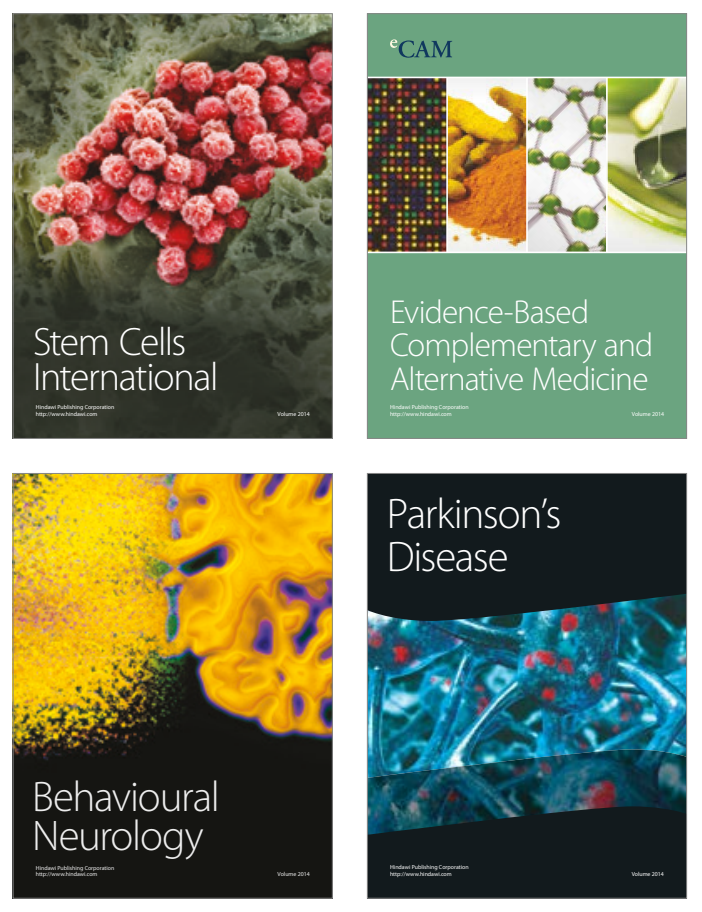
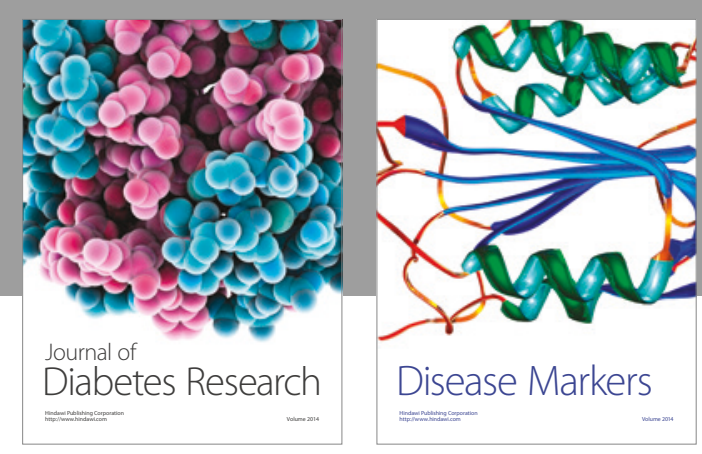

Disease Markers
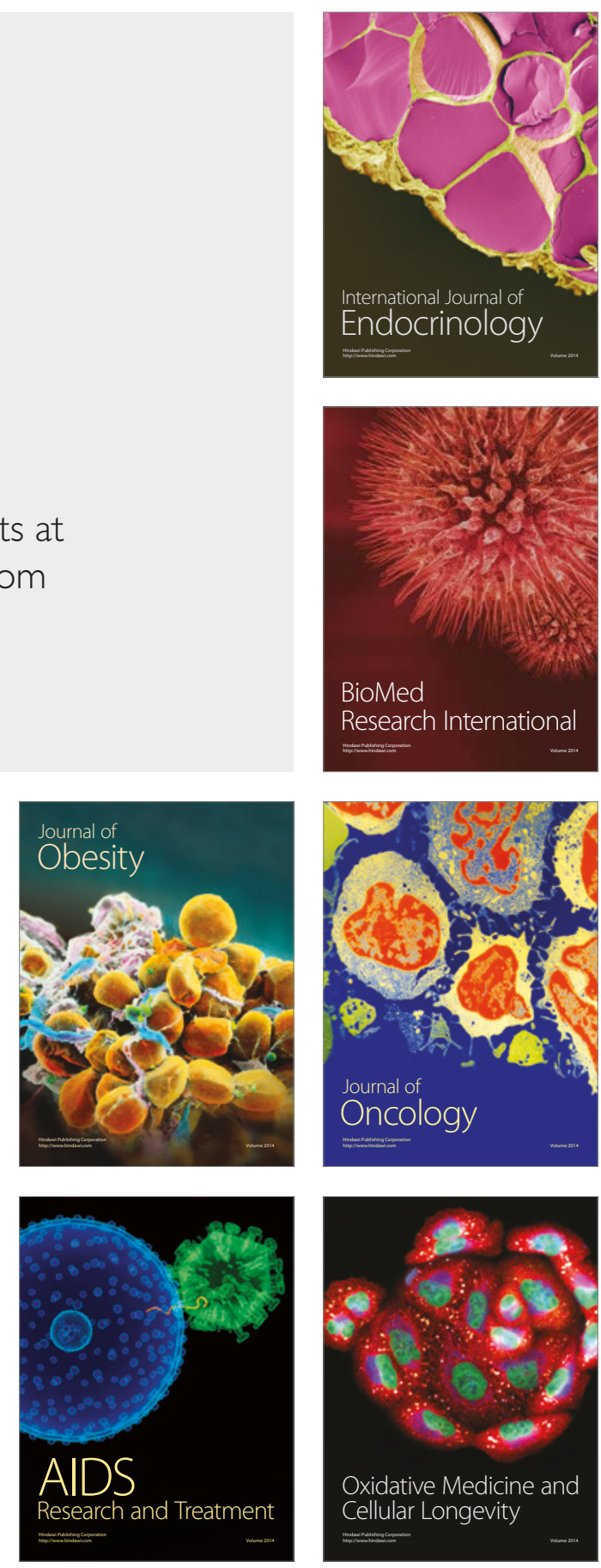